\title{
3D Stereolithographic Modeling Technique for Hemimandibular Reconstruction Report of a Case with Innovation Technique
}

\author{
Pao-Hsin Liu' ${ }^{1}$, Tong-Yui V Wong ${ }^{2,3}$, Jing-Jing Fang ${ }^{4}$, Ken-Chun Chen ${ }^{2}$, Jenn-Ren Hsiao ${ }^{5}$, \\ Jehn-Shyun Huang ${ }^{2,3, *}$ \\ ${ }^{1}$ Department of Biomedical Engineering, I-Shou University, Kaohsiung, Taiwan \\ ${ }^{2}$ Department of Stomatology, National Cheng Kung University Hospital, Tainan, Taiwan \\ ${ }^{3}$ Institute of Oral Medicine, College of Medicine, National Cheng Kung University, Tainan, Taiwan \\ ${ }^{4}$ Department of Mechanical Engineering, National Cheng Kung University, Tainan,, Taiwan \\ ${ }^{5}$ Department of Otolaryngology, National Cheng Kung University Hospital, Tainan, Taiwan \\ *Corresponding Author: huangjs@mail.ncku.edu.tw
}

Copyright (C 2014 Horizon Research Publishing. All rights reserved.

\begin{abstract}
Computer aid design and manufacture have been widely applied in oral and maxillofacial surgery. Reconstruction of hemi-mandibular defects after tumor ablation is a major challenge for clinicians due to its complex architecture. We report of a case treated with innovation technique, including the use of computer assisted modeling to preoperatively design of the excision of the mandible tumors and the integration of rapid prototyping techniques in mandibular reconstruction. The patient received hemimandibulectomy for the tumor excision and reconstructed with titanium condyle-plate, and followed up for 4 years without recurrence and retained good function.
\end{abstract}

\begin{tabular}{llll} 
Keywords & \multicolumn{2}{c}{ Hemimandibulectomy, } & Mandible \\
Reconstruction, & Virtual & Surgical & Planning, \\
Stereolithographic Biomodelling & &
\end{tabular}

\section{Introduction}

The mandible, the largest and strongest bone of the face, serves for the reception of the lower teeth. It consists of a curved, horizontal portion, the body, and two perpendicular portions, the rami, which unite with the ends of the body nearly at right angles. The mandible articulates with the skull base with the temporomandibular joints (TMJ) bilaterally. The TMJ is a load-bearing articulation that is connected to its contralateral counterpart by a single bone (i.e., the mandible connects with the cranium through both TMJs). The mandible is also one of the most important skeletons to maintain the harmony and balance of facial profile.

In case of patients with huge and aggressive jaw bone tumor would be allocated for mandibular resection with plate reconstruction and subsequent living fibular bony tissue transfer $[1,2]$. It is very difficult to reshape or restore the mandible when it has been resected due to the complex architecture of the mandible and the unique articulation of bilateral TMJs. Post-operative disfiguration is often encountered when the defect is large and the mandible is discontinuous [3]. Stereolithographic biomodelling is a technology that allows three-dimensional computed tomography data to be used to manufacture solid plastic replicas. These 3D models can be used to pre-shape metallic implant that can be used in surgery. The length of implant plates and the number and lengths of required screws can be planned before surgery [4].

Traditionally, the pre-surgical stereolithographic biomodel was made of the replica of the deformed jaw bone disfigured by the huge tumor. The surgeons have to sculpture the original model into the harmonious or symmetrical ones. With the aid of virtual method, the diseased part of the mandible can be replaced by non-diseased part by mirror imaging technique and create a symmetrical stereolithographic biomodel for surgical planning. This modified virtual method has been reported to be useful and effective in surgical reconstruction after mandibular tumor ablation and especially for the huge defect involving the midline of mandible [5].

When the segmental resection involving the mandibular condyle, the technique become more complex [6]. Hanasono \& Skoracki, (2010) suggested that virtual planning and stereolithographic biomodel may increase the accuracy of reconstruction, especially in the restoration of the anterior curvature and mandibular angle, and in re-establishing the correct height of the ramus/condyle. Good dental occlusion could be achieved in $90 \%$ patients with this method. Of patients with malocclusion post-operatively, all had undergone resection of one temporomandibular joint [7].

The current study reported a case of ameloblastoma, a 
benign but aggressive invasion tumor, in the right mandible including the condyle. The patient received hemimandibulectomy for the tumor excision (from midline symphysis to the condyle) and reconstructed with titanium condyle-plate. There are very few papers discussed the reconstruction of mandible after hemimandibulectomy, especially when the tumor involves the condyle of mandible. We describe a novel technique of presurgical planning with the 3D virtual method with $\mathrm{CT}$ scan images and stereolithographic biomodelling.

\section{Materials and Methods}

\section{Report of the Case}

A 42-year-old male was referred to NCKU medical center in March 2009 due to right facial swelling for 5-6 years (Figure 1). He has been to a local hospital for consultation. But he did not take action for the treatment until now. Recently, he noted the tumor grew and enlarged rapidly. He sought help in a local dental office and was referred to our department for further evaluation and treatment. He received biopsy on the day presented and proved to be Ameloblastoma (Figure.1). Panoramic x-ray and computer tomograme revealed the tumor affected the right half of the mandible upto the subcondylar region (Figure 2).

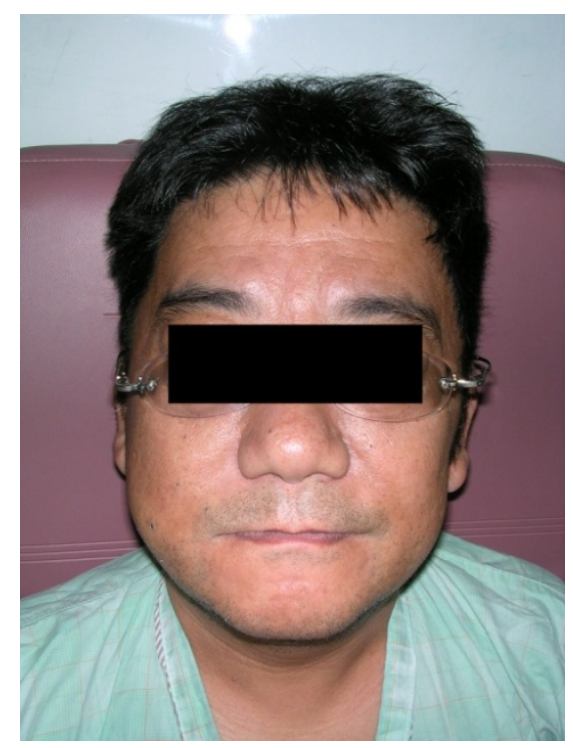

Figure 1. The facial profile at the initial presentation notes the swelling over the right mandible.

\section{Preoperative Virtual Planning}

Mirror imaging coupled with laser stereolithographic model

An anatomically exact Stereolithographic (SLA) model of the jaws based upon preoperative computed tomography (CT) scan data is produced (Figure 3). The process involves CT scan, computer technique, and acrylic photo-polymerizing resin [8]. A virtual method is developed in the Virtual Reality Laboratory in National Cheng Kung University in collaboration with department of Oral and Maxillofacial Surgery for 3D visualization and manipulation of the reformed 3D CT data [9]. The processing tools used include segmentation, regional growing, pasting, erasing, drawing, and mirrowing.

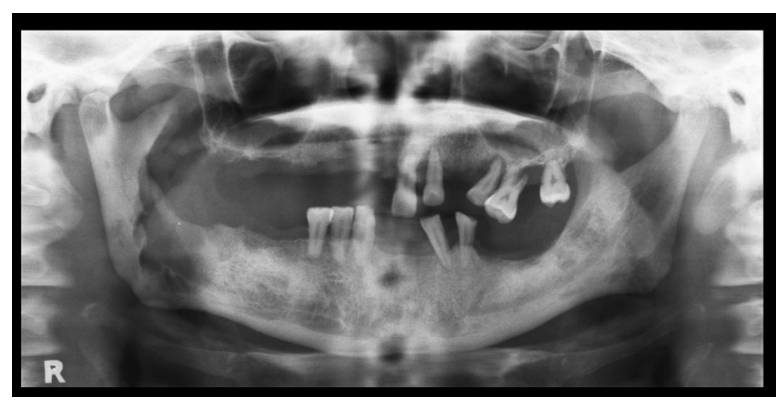

Figure 2. The panoramic X-ray taken before the operation. Notes the massive bony destruction over the right mandible involving the ramus and condylar head.

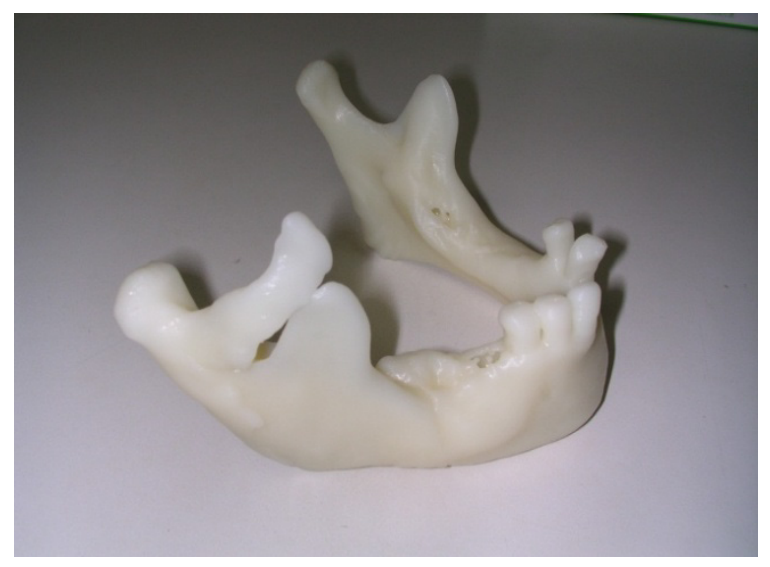

Figure 3. A $3 \mathrm{D}$ stereolithographic modeling constructed with mirror image of the opposite mandible (non-disease half) is served for pre-surgical planning and plate bending..

Preoperatively, spiral computed tomography (CT) scanning of the facial skeleton (upto the cranial base) was done using $3 \mathrm{~mm}$ slice thickness. The CT scanning is taken with a $3-\mathrm{mm}$ table increment interpolated to form a $1-\mathrm{mm}$ slice thickness. The acquired digital data (DICOM) were transferred to the computer to generate the surgical planning. For reconstruction of hemimandibulectomy, the diseased portion on the image of mandible was identified and erased. The counterpart structure on the normal side could be extracted, mirrored the image, and superimposed on the defect. The condyle cap was retained as a guide post to ensure the accurate position of the construction [8]. The mirror image on the mandible was adjusted to match the primary mandible remnant until the maximal degree of merging was achieved.

\section{D Stereolithographic Biomodeling Technique}

The CT data are transferred to a workstation in the Virtual 
Reality Laboratory, and then programmed to a file readable by the stereolithography machine (STL format) with a preceding modification [5, 9]. This biomodel served as a guide for surgical planning. A reconstruction plate was bent and adapted to fit the contour of the model. The patient with ameloblastoma in the right mandible received tumor excision including hemimandibulectomy and reconstructed with titanium condyle-plate. This protocol was approved by the ethical committee of the National Cheng Kung University Hospital in Tainan (Taiwan). And the patient gave his consent to receive such a surgical therapy. Patient was followed up for 4 years without recurrence and regained good harmony facial profile and jaw bone function.

\section{Results}

Due to the disease entity of ameloblastoma is an aggressive invasion tumor. The operation was recommended as tumor excision including right side of mandible and condyle, and then reconstructed with the free fibular myocutaneous flap and reconstructive titanium plate with condylar prosthesis. But the patient could not afford the treatment and hospital stay due to personal reasons. He deferred the treatment and asked for the other option. Therefore, the treatment plan of tumor excision with hemimandibulectomy and reconstructed with the Ti-reconstruction plate were suggested. Staged reconstruction with bone graft was also recommended in 2 months after the surgery. The patient preferred the latter option of the treatment plan and received the surgery on Apr. 2009. The patient underwent the hemimandibulectomy for tumor excision and reconstructed with the reconstruction plate with condylar head. The prebend plate and condyle fit in the mandibular defect perfectly without any adjustments. The patient recovered uneventfully and follow-up until now for 4 years without recurrence or complications (Figure 4 and 5). He did not received the bone graft for the rehabilitation of the dentures. Patient can open his mouth for $30 \mathrm{~mm}$ without TMJ pain after surgery.

\section{Discussion}

The current study reported a case of ameloblastoma, a benign but aggressive invasion tumor, in the right mandible which received tumor excision including hemimandibulectomy and reconstructed with titanium condyle-plate. Patient was followed up for 4 years without recurrence and regained good harmony facial profile and jaw bone function.

The remnant mandibular fragments tend to become freely mobile following segmental mandibulectomy. Traditional techniques to translate the straight reconstruction plate into the curved shape of the mandible and positioning the artificial condyle in the TMJ fossa allow for proper condylar positioning have often been imprecise. A method is presented for the prefabrication and fitting of metal reconstruction plates that overcomes this difficulty and also enables improved preoperative planning of microvascular free flaps.

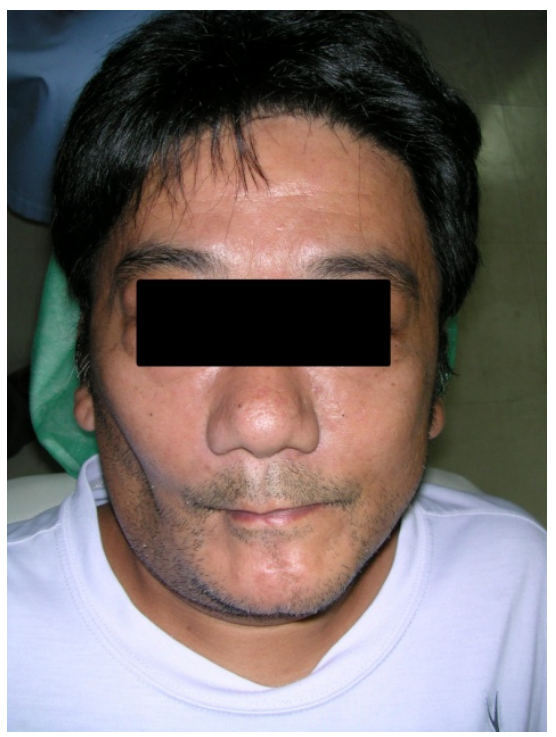

Figure 4. The facial profile of the patient 4-year after the surgery.

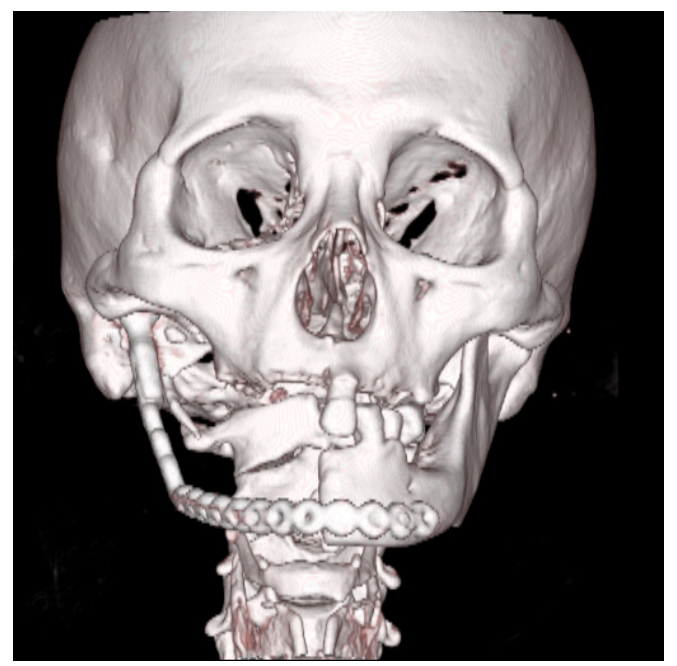

Figure 5. Post operative 4 years, reconstructive computer tomogram of mandible shows symmetrical facial profile and the right metal condylar head seated in the fossa properly.

The advantages of this method presented are preoperative diagnosis and planning, with direct visualizing the codylar position in the glenoid fossa, and 3D biomodel for prebending the reconstruction plate. This technique significantly improves the predictability of clinical outcome when compared with similar treatments without its use. And this technique had been claimed to have the benefits of reducing the total operation time [10]. Therefore, it had the benefit of decreasing the general anesthesial time and reducing the wound exposure time. It also provides the tool for multidiscipline communication and patient education.

Although, this presurgical stereolithographic modeling technique provides many advantages, there are still some 
shortages claimed. This techniques need cooperation between the surgeons, computer technicians and the stereolithographic manufacturers. It takes about one week for the model construction, and it also cost an extra payment for the patient. For the convetional alloplatic method, it does not need any cost or time for the preoperative stage. But the surgeons have to contouring the plate during the surgery. Generally, it takes about one more hour for the free hand bending the plate and fixation. The result of facial profile may not esthetic acceptable, if the surgeon is not well trained or experienced. Comparing with the conventional alloplastic procedure, the stereolithographic modeling facilitates the surgical procedure and accomplishes an esthetic profile.

The computer-simulated surgery is helpful in mandibular reconstructions after (1) tumor-induced deformation, (2) extensive bony destruction, or (3) nonstabilized defects resulting in displacement of the resection stumps [11]. The 3D model allowed the surgeon to reduce the dislocated fragments with correct condyle positioning and midline alignment and to pre-bend and adapt the reconstructive (osteosynthesis) plate.

Computer-aided surgery has become clinical usage and preoperative virtue planning is popular in the last decade. Technical progress led to the integration of rapid prototyping techniques (3-dimensional stereolithography) in mandibular reconstruction after tumor ablation. There are no differences in the accuracy of virtual three-dimensional reconstructions of human dry mandibles, produced with the surface processing protocols (smoothing or refinement) [12]. Lethaus et al. (2012) found the pre-bend plate was used in all patients intraoperatively without the need for any further bending [13].

Although, the mandibular reconstruction after tumor ablation using a vascularised fubular flap is current is current a standard treatment option [14]. Sometimes, the patient may have difficulties to have a multiple detrimental procedures in one appointment and defer for a staged surgery for reconstruction. The use of vascularised bone tissue in secondary reconstruction permits superior restoration of articulation, deglutition and mastication; aesthetic improvement of facial appearance; and improvement of the patient's quality of life in comparison with non-vascularised alternatives [15].

Marunick et al. (1992) evaluate the masticatory function of 5 hemimandibulectomy patients. And they found that the extent of mandibular resection and loss of continuity tend to decrease masticatory function [16]. Mandibular continuity defects are now usually reconstructed with vascularised fubular flap. However, factors associated with the tumor and the patient can still be reasons to choose the reconstruction plates [17]. Okura et al. (2005) conducted a retrospective analysis of 100 consecutive patients who underwent immediate bridging plate reconstruction and found a good long term survival and favorable function [18]. We describe a novel technique of presurgical planning with the $3 \mathrm{D}$ virtual method with CT scan images and stereolithographic biomodeling. The patient reconstructed the mandible with a reconstruction plate for 4 years with acceptable jaw function.

Most literature reiterates established reconstructive techniques including the use of computer assisted modeling to preoperatively design of the excision of the mandible tumors and the integration of rapid prototyping techniques in mandibular reconstruction [2]. Although CAD/CAM has been extensively used in many aspects of the medical field, its application in reconstruction of half mandible defects (including the condyle) should be further investigated.

\section{Conclusion}

Reconstruction of hemi-mandibular defects after tumor ablation is a major challenge for clinicians due to its complex architecture and free movable remnant segment. In this case reported, we presented a novel technique of presurgical planning with the $3 \mathrm{D}$ virtual method and stereolithographic biomodelling. The advantages of this method are preoperative diagnosis and planning, with direct visualizing the codylar position in the glenoid fossa, and 3D biomodel for prebending the reconstruction plate. This technique significantly improves the predictability of clinical outcome.

\section{Acknowledgements}

The authors thanks to the generous work and help from the Biomechenics Lab of Professor Jin-Jin Fang and Tong-Yue Wong for the surgical stereolithographic model construction.

\section{Conflict of Interests}

The authors declare that there is no conflict of interests regarding the publication of this article.

\section{REFERENCES}

[1] B. C. Kim, S Kim, W Nam, I. H. Cha, H. J. Kim. Mandibular Reconstruction with Vascularized Osseous Free Flaps: a Review of the Literature. Asian Pacific J Cancer Prev. 2012; 13: 553-558.

[2] R. E. Hayden, D. P. Mullin, and A. K. Patel. Reconstruction of the segmental mandibular defect: current state of the art. Curr Opin Otolaryngol Head Neck Surg 2012; 20: 231-236.

[3] G. Wang, J. Li, A. Khadka, Y Hsu, W Li, J Hu. CAD/CAM and rapid prototyped titanium for reconstruction of ramus defect and condylar fracture caused by mandibular reduction. Oral Surg Oral Med Oral Pathol Oral Radiol. 2012; 113: 356-361.

[4] A. Cohen, A. Laviv, P. Berman, R. Nashef, J. Abu-Tair. Mandibular reconstruction using stereolithographic 3-dimensional printing modeling technology. Oral Surg Oral Med Oral Pathol Oral Radiol. 2009; 108: 661-666.

[5] T. Y. Wong, J. J. Fang, C. H. Chung, J. S. Huang, J. W. Lee. 
Comparison of two methods of making surgical models for correction of facial asymmetry. J Oral Maxillofac Surg 2005; 63:200-208.

[6] M.A. Pogrel, S. Podlesh, J.P. Anthony, J. Alexander. A comparison of vascularized and nonvascularized bone grafts for reconstruction of mandibular continuity defects. J Oral Maxillofac Surg 1997; 55: 1200-1206.

[7] M. M. Hanasono, J. P. Zevallos, R. J. Skoracki, P. Yu. A Prospective Analysis of Bony versus Soft-Tissue Reconstruction for Posterior Mandibular Defects. Plast Reconstr Surg 2010; 125: 1413-1421.

[8] J. W. Lee, J. J. Fang, J. R. Chang, C. K. Yu. Mandibular defect reconstruction with the help of mirror imaging coupled with laser stereolithographic modeling technique. J Formos Med Assoc 2007; 106: 244-250.

[9] T. Y.Wong,J. J. Fang, C. H. Chung, J. S. Huang. Restoration of the temporal defect using laser stereolithographic technique. J Oral Maxillofac Surg 2002; 60:1374-1376.

[10] P Mehra, J Miner, R D'Innecenzo, M Nadershah. Use of 3-D stereolithographic models in oral and maxillofacial surgery. $\mathrm{J}$ Maxillofac Oral Surg 2011; 10: 6-13.

[11] J. P. Levine, A. Patel, P. B. Saadeh, D. L. Hirsch. Computer-aided design and manufacturing in craniomaxillofacial surgery: The new state of the art. J Craniofacial Surg 2012; 23: 288-293.

[12] E. Gomes Ferraz, L. C. S. Andrade, A. R. dos Santos, V. R.
Torregrossa, M. do R. S. Freire, V. A. Sarmento. Effect of different surface processing protocols in three-dimensional images for rapid prototyping. Advances in Engineering Software 2011; 42: 332-335.

[13] B. Lethaus, L. Poort, R. Böckmann, R. Smeets, R. Tolba, P. Kessler. Additive manufacturing for microvascular reconstruction of the mandible in 20 patients. $\mathrm{J}$ Cranio-Maxillo-Facial Surg 2012; 40: 43-46.

[14] L. Ciocca, S. Mazzoni, M. Fantini, F. Persiani, P Baldisara, C. Marchetti, R. Scotti. A CAD/CAM-prototyped anatomical condylar prosthesis connected to a custom-made bone plate to support a fibula free flap. Med Biol Eng Comput 2012; 50: 743-749.

[15] T. A. Iseli, J. C. Yelverton, C. E. Iseli, W. R. Carroll, J. S. Magnuson, E. L. Rosenthal. Functional Outcomes Following Secondary Free Flap Reconstruction of the Head and Neck. Laryngoscope 2009; 119: 856-860.

[16] M. T. Marunick, B. E. Mathes, B. B. Klein. Masticatory function in hemimandibulectomy patients. J Oral Rehabil 1992; 19: 289-295.

[17] B. van Minnen, J. M. Nauta, A. Vermey, R. R. M. Bos, J. L. M. Roodenburg. Long-term fuctional outcome of mandibular reconstruction with stainless steel AO reconstruction plates. Br J Oral Maxillofac Surg. 2002; 40: 144-148.

[18] M. Okura, E. T. Isomura, M. Kogo. Long-term outcome and factors influencing bridging plates for mandibular reconstruction. Oral Oncology 2005; 41: 791-798. 